Military Technical College Kobry El-Kobbah, Cairo, Egypt

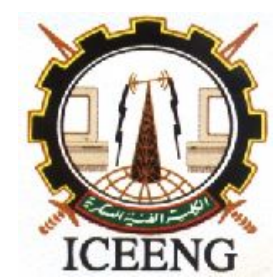

\section{$9^{\text {th }}$ International Conference on Electrical Engineering ICEENG 2014}

\title{
Simulation Study of the Effect of Chopping Jamming on LFM Pulse Compression Radar
}

\author{
$\underline{B y}$ \\ A. Abu El-Fadl Fathy M. Ahmed Mohamed Samir A. Alaudeen H. Asseesy*
}

\section{Abstract:}

Linear frequency modulated pulse compression (LFM-PC) radar is one of the widely used modern radar systems. It has high immunity against conventional noise jamming due to its high processing gain. To sufficiently affect such radar, smart jamming techniques should be applied. In the last decade, different smart jamming techniques have been proposed. However, literature lacks to the analysis and evaluation of the effect of such techniques on modern radar systems. In this paper, the effect of one of the recently proposed jamming techniques, called chopping jamming, is analyzed and evaluated against LFM-PC radar depending on a typical LFM-PC verified radar model. The worthy of the application of chopping jamming on LFM-PC radar regarding to the probability of false alarm as well as radar detection is discussed and presented.

\section{Keywords:}

LFM, PC, Chopping jamming.

\section{Introduction:}

The contradiction between long detection range and high range resolution is resolved using pulse compression techniques[1]. Linear frequency modulation is widely used with high speed targets due to its tolerance to high Doppler shifts[2].

To mask the target, conventional noise jamming aims to degrade Signal-to-Noise Ratio (SNR) at the input of the detector by applying noise power at the radar receiver frontend. The power level of the noise should exceed that of received radar signal. The

* Egyptian Armed Forces

difference between jamming signal power and received radar signal power, known as 
Jamming-to-Noise Ratio (JSR), is in the order of $6 \mathrm{~dB}$ for a conventional radar[3]. Concerning modern LFM PC radar, the jammer should increase its JSR to compensate for the processing gain provided by pulse compression and coherent integration[4]. The gain provided by pulse compression is equal to time-bandwidth product of the radar pulse[5]. Coherent integration gain depends on the Coherent Pulse Interval (CPI) of the radar processor. Typically, the total processing gain can take values between 20-50 dB [6]. This implies a very high power level of jamming signal, which, in most cases, cannot be achieved.

This power budget problem leads to the need for smarter jamming signals that need less power levels. The main trend to jam LFM PC search radars is to distort the output of radar matched filter and, consequently, affect radar detection without excessive power levels. In the literature, there are different published jamming techniques with the aim of distorting radar matched filter output. The common target of these techniques is to generate spurious signals in the output of the matched filter in addition to the real signal to limit the performance of the range tracker [7-10].

One way to distort the radar matched filter output, as proposed in [11], is by using chopping jamming that depends on extracting predetermined segments at different time slots from the radar pulse, each segment is repeated till the following segment and so on. This jamming pulse has the same pulse width as the radar pulse but with different carrier modulation. Although this jamming technique, like others, has been proposed to affect LFM-PC radar range tracker, its effect on radar detection is investigated in the present work. In other words, chopping jamming has been proposed to affect tracking radar, but its effect on LFM-PC search radars is examined here.

Following this introduction, this paper is organized as follows: section 2 introduces both mathematical and simulation radar model for LFM PC radar. This model is based on previous work published earlier in [12] for the authors. After verification of the model, in section 3, chopping jamming technique is modeled. The performance of LFM PC radar in presence of chopping jamming is analyzed in section 4 . The performance analysis is based on investigating the radar probability of detection versus SNR at different JSR values of the proposed model. Conclusion and comments on the results are provided in section 5 .

\section{LFM-PC Radar Model:}

The radar model of LFM PC radar, shown in Figure (1), is the same introduced and verified by the authors in [12]. So, only key parameters and block diagram of the model are revisited here. The complex envelop of LFM PC radar pulse with bandwidth $B$ and pulse width $T$ is[13]: 


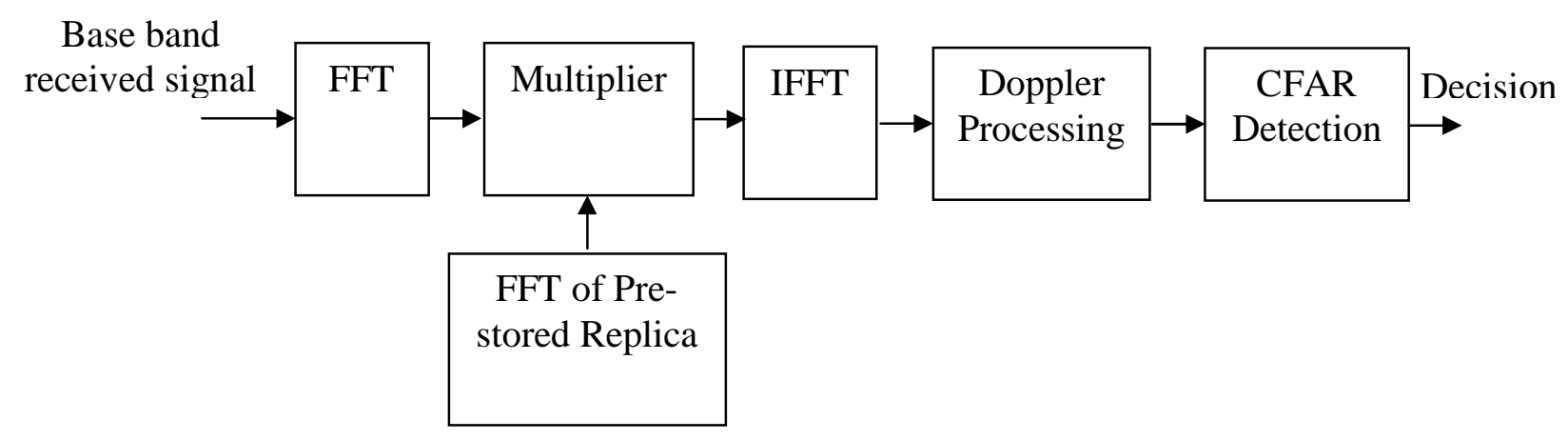

Figure (1): Block diagram LFM PC radar model.

$$
s(t)=\frac{\mathbf{1}}{\sqrt{T}} \operatorname{rect}\left(\frac{t}{T}\right) \exp \left(j \pi k t^{2}\right) \quad k= \pm \frac{B}{T}
$$

The matched filter output magnitude response due to the LFM signal of Equation (1) can be calculated by performing a convolution process between this signal and the matched filter impulse response and is given by [5, 14]:

$$
|y(t)|=\operatorname{sinc}\left(\pi B\left(t-T-t_{d t}\right)\right)
$$

Where ${ }^{t_{d t}}=\frac{2 R_{\tau}}{C}, \mathrm{R}_{\mathrm{t}}$ is the target range and $c$ is the speed of light.

For LFM-PC radar, the additional processing gain due to pulse compression and coherent integration are added to the term SNR $[6,15]$,so that the new SNR becomes:

$$
S N R_{z}=S N R \cdot N \cdot B T
$$

Where, SNR is the peak signal power to average-noise power ratio for a conventional radar, $\mathrm{N}$ is the number of pulses in one CPI and BT is the compression gain. Hence, detection probability of pulse compression radar, $P_{d \mathbf{z}}$, based on Nyman-Pearson criteria, is given by[15]:

$$
P_{d \mathbf{z}}=Q\left(\sqrt{2 S N R_{\mathbf{z}}}, \quad \sqrt{2 \ln \left(\frac{\mathbf{1}}{P_{f a}}\right)}\right)
$$

Where $\mathrm{P}_{\mathrm{fa}}$ is the probability of false alarm and $Q(\mathrm{O})$ is the Marcum function.

The assumed simulated radar and target parameters are shown in Table (1). Radar parameters have been chosen to simulate typical pulse compression search radar[6]. Whereas the chosen target parameters allow the target to be within a single range cell and a single Doppler cell to prevent range or Doppler straddle[12].

The simulated and the theoretically derived detection curves are calculated in clear environment and shown in Figure (2). The simulated and the theoretical results agreed very well to each other[12]. 
Table (1). Radar and Target Parameters

\begin{tabular}{|l|c|c|}
\hline Radar Parameter & Value & Units \\
\hline Pulse Width Repetition & 10 & $\mu_{\mathrm{s}}$ \\
\hline $\begin{array}{l}\text { Pulse } \\
\text { Interval }\end{array}$ & 1.6 & $\mathrm{~ms}$ \\
\hline Carrier Frequency & 3 & $\mathrm{GHz}$ \\
\hline Chirp Bandwidth & 7.576 & $\mathrm{MHz}$ \\
\hline Target Range & 312 & $\mathrm{Km}$ \\
\hline Target Doppler & \multicolumn{2}{|c|}{ Cell Average } \\
\hline CFAR Type & 16 & $\begin{array}{c}\text { Range } \\
\text { cells }\end{array}$ \\
\hline CFAR Window size & \multicolumn{2}{|c}{} \\
\hline
\end{tabular}

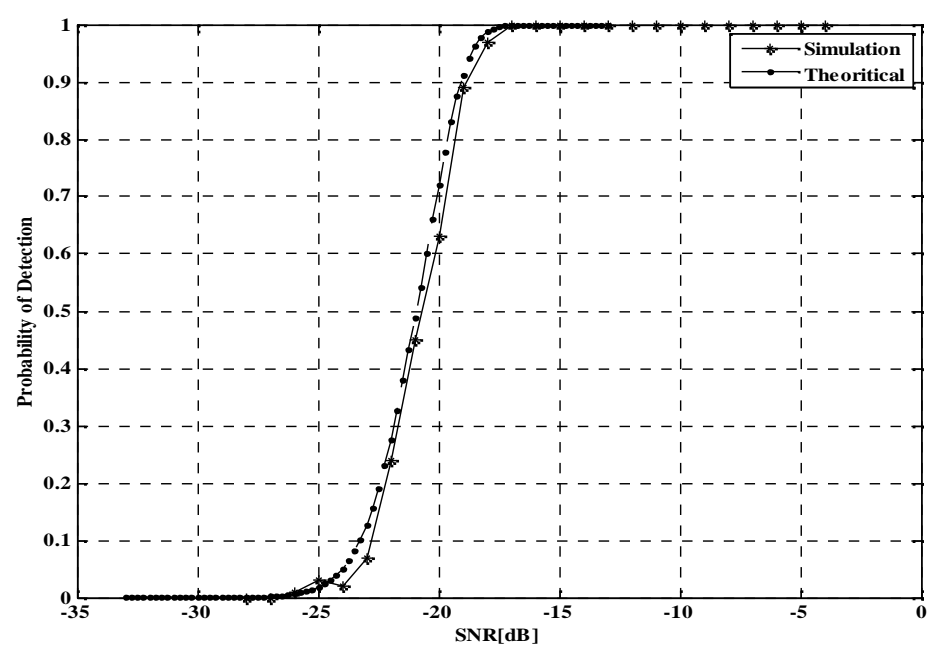

Figure (2): Simulated and derived detection curves for LFM PC radar in clear environment at $\mathrm{P}_{\mathrm{fa}}=10^{-7}$.

\section{Chopping Jamming Model:}

In accordance with [11], chopping jamming is used by a Digital Radio Frequency Memory (DRFM) based airborne repeater jammer . Digital radio frequency memory (DRFM) is a technique in which high speed sampling and digital memory are used for storage of radio frequency and microwave signals. DRFM is widely used in Electronic Counter Measures (ECM) applications especially when coherent jamming techniques are to be generated[16-20].

In a DRFM, the input RF is first down-converted in frequency, and the sampled with a high-speed analog-to digital converter (ADC). The samples are stored in memory and they can be manipulated in amplitude, frequency and phase to jam the radar signal. The stored samples are later recalled from memory, processed by the digital to-analog 
(DAC), up-converted and transmitted back to the radar[21, 22].

From the output samples, predetermined segments of the LFM pulse are stored in a memory. From these segments, the jamming pulse is formed by repeating each stored segment for a predetermined number of times and concatenating the segments and their copies adjacent to each other preserving the original pulse width. The baseband jamming pulse can be expressed mathematically as follows:

$$
J(t)=A \sum_{i=1}^{M} \sum_{i=1}^{N} \exp \left(j k_{j} t_{l i}^{2}\right)
$$

Where A is the pulse amplitude, $M$ is the total number of segments, $N$ is the number of repetitions of each segment, $\mathbf{k}_{\mathbf{j}}$ is the segment frequency slope and it is equal to $k / \mathrm{N}$, and $t_{l i}$ is the time vector along an individual segment. It can easily be found that the segment width is:

$$
T_{\text {segment }}=\frac{T}{M \cdot N}
$$

To evaluate the effect of chopping jamming on the verified LFM PC radar model introduced in section II in this paper, a Matlab simulation model for chopping jamming has been built.

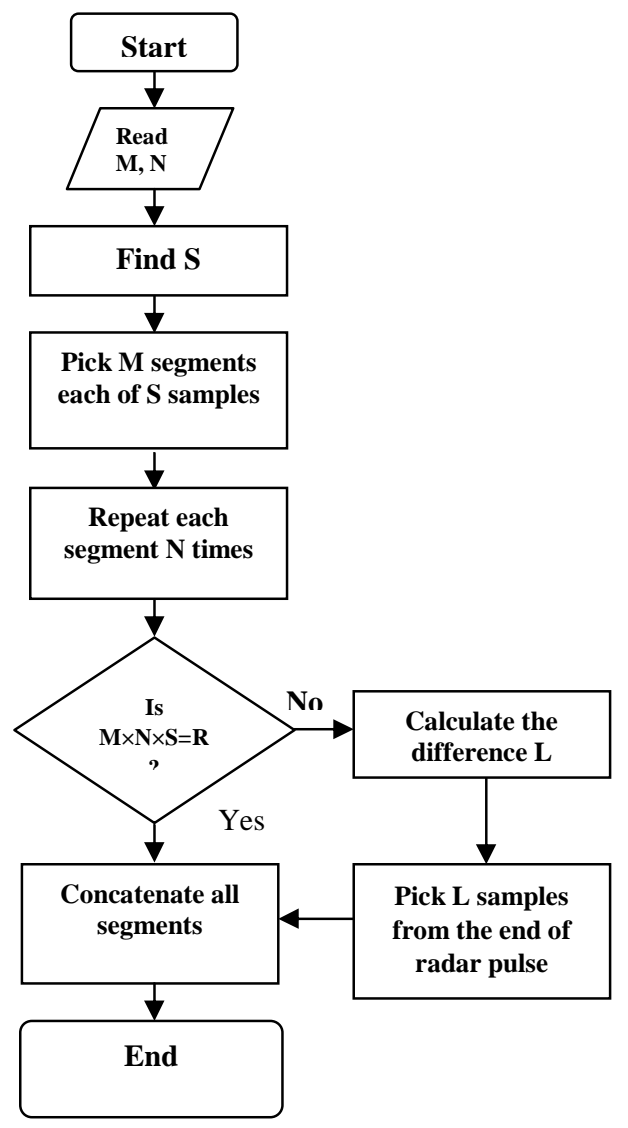

Figure (3): Flow chart of chopping jamming simulation model. 
As shown in Error! Reference source not found.), given the received radar pulse, the model acquires the number of required segments and the number of repetitions of each segment. The program calculates the positions and number of samples of each segment $S$. The required segments are then picked from the radar pulse, repeated as required, and then each segment along with its repetitions is concatenated with each other. If $M \times N \times S$ is less than the total number of samples $R$ within the received radar pulse, the model calculate the difference $L$ and fill the last part of the jamming pulse with the last $L$ samples in the received pulse.

The generated signal by this simulation model, shown in Figure (4.b), agreed with the jamming waveform introduced in [11].

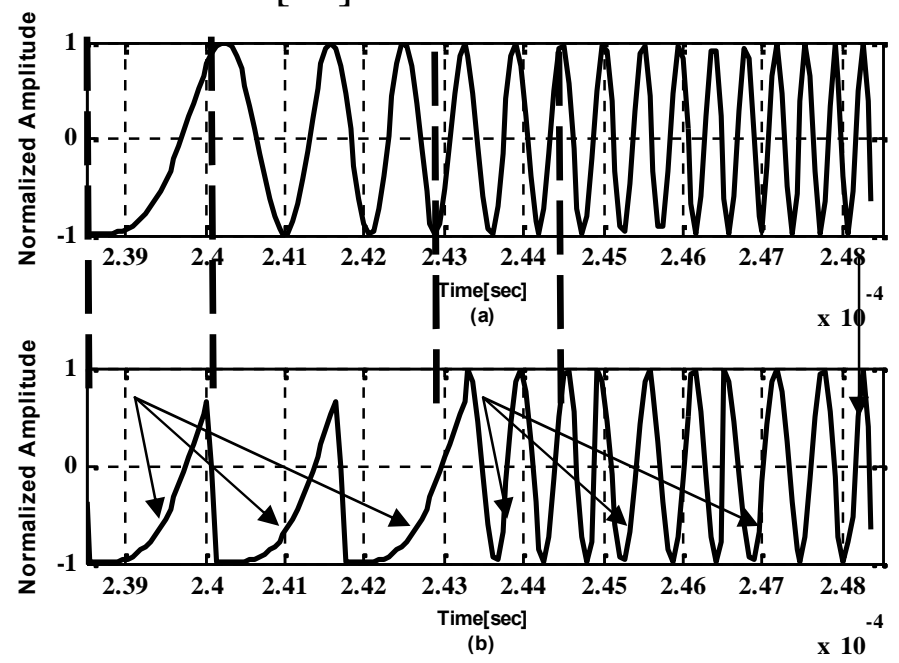

Figure (4): Time domain representation of (a) radar signal and (b) chopping jamming $\operatorname{signal}(M=2, N=\mathbf{3})$.

\section{Performance analysis of LFM-PC radar in presence of chopping jamming:}

After building both radar and jamming models, the effect of chopping jamming on LFM PC radar is to be investigated. In this investigation, the jammer delay with respect to the radar return is assumed $100 \mathrm{~ns}$, which is the typical-worst case delay by state of the art DRFM. In accordance with Error! Reference source not found.), the output of the IFFT block represents the radar matched filter output. This is the first output to be evaluated.

Figure (5) shows the output of matched filter in presence of chopping jamming at $\mathrm{JSR}=0 \mathrm{~dB}$. The figure shows the true target along with numerous range false targets (RFT) with lower level. The loss of power in false targets relative to true target is due to the mismatch between radar signal and jamming signal[11]. 
Up to this point, the results found by Sparrow in [11] are verified and the effect of chopping jamming on LFM PC matched filter proposed in this paper agreed with Sparrow's results.

Sparrow aimed to affect range-tracking radar by generating RFTs at the output of the matched filter. In the present work, it is required to investigate the effect of Sparrow's technique on the radar detection. To do this, according to Figure (1), the output of the matched filter is passed through a coherent integrator with gain $12 \mathrm{~dB}$ then it is passed through a frequency domain CFAR processor with the parameters listed in Table (1). This model is consistent with modern search radar systems.

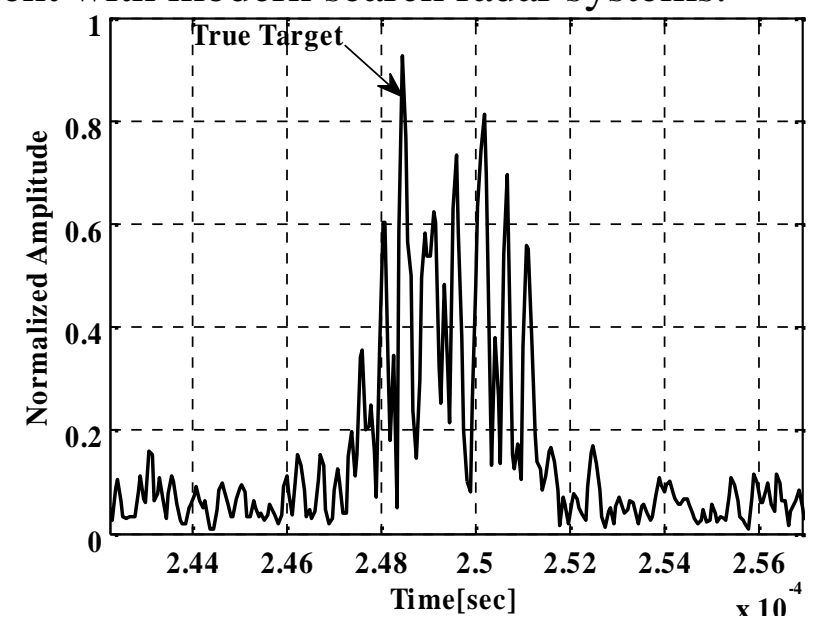

Figure (5): LFM-PC Matched filter output at JSR=0dB and $\mathrm{SNR}=-5 \mathrm{~dB}$ with chopping jamming.

As stated in [11], the jammer is assumed to be a Self Screening Jammer (SSJ). This implies that the received jamming signal by the radar has the same Doppler as the target[23]. The target Doppler shift is lost if any other configuration of the jammer (Stand Off Jammer (SOJ) or Stand-Forward Jammer (SFJ)) is used. In this analysis, the two scenarios are to be tested.

In SSJ case, with a jamming signal has the same Doppler shift as the target return, the jamming signal covers the target signal at the output of the coherent integrator as shown in Figure (9). 


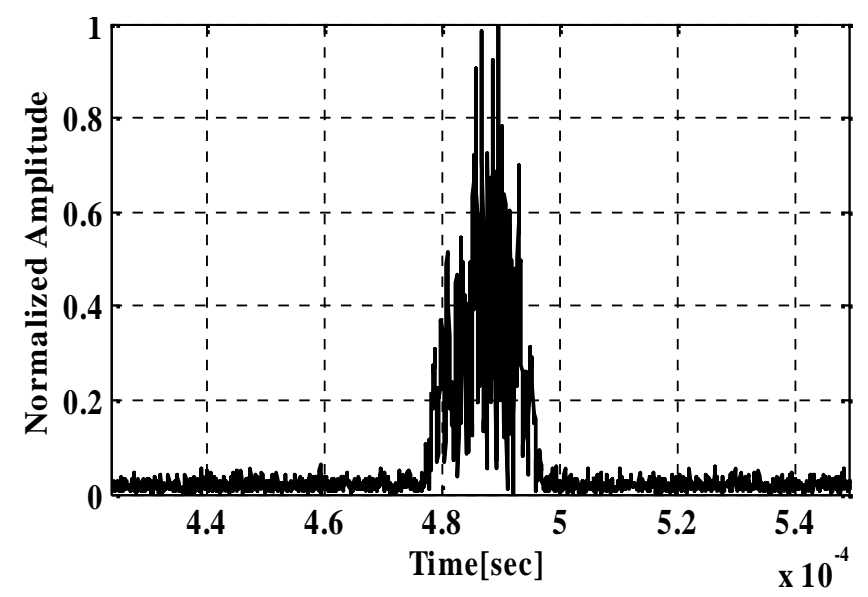

Figure (6): SSJ Generated RFTs at the output of coherent integrator at the target Doppler cell with JSR $=10 \mathrm{~dB}$ and $\mathrm{SNR}=-5 \mathrm{~dB}$.

These RFTs at the output of the coherent integrator causes the CFAR threshold to be raised, as shown in Figure (7), causing misdetection.

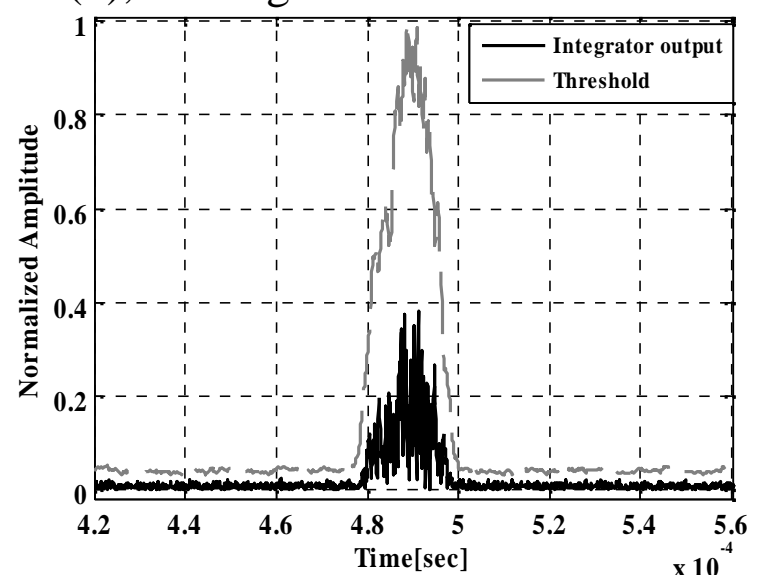

Figure (7): $C F A R$ threshold with $\mathrm{JSR}=10 \mathrm{~dB}$ and $\mathrm{SNR}=-5 \mathrm{~dB}$ at target Doppler cell with SSJ.

However, if the jammer is an SOJ or an SFJ jammer with no information about the target Doppler shift, the jamming signal lies in the zero-Doppler cell as shown in Figure (8). Therefore, the target Doppler cell is free from any RFTs. Figure (9) exhibits the ability of coherent integrator to cancel the effect of all RFTs generated by an SOJ or an SFJ. 


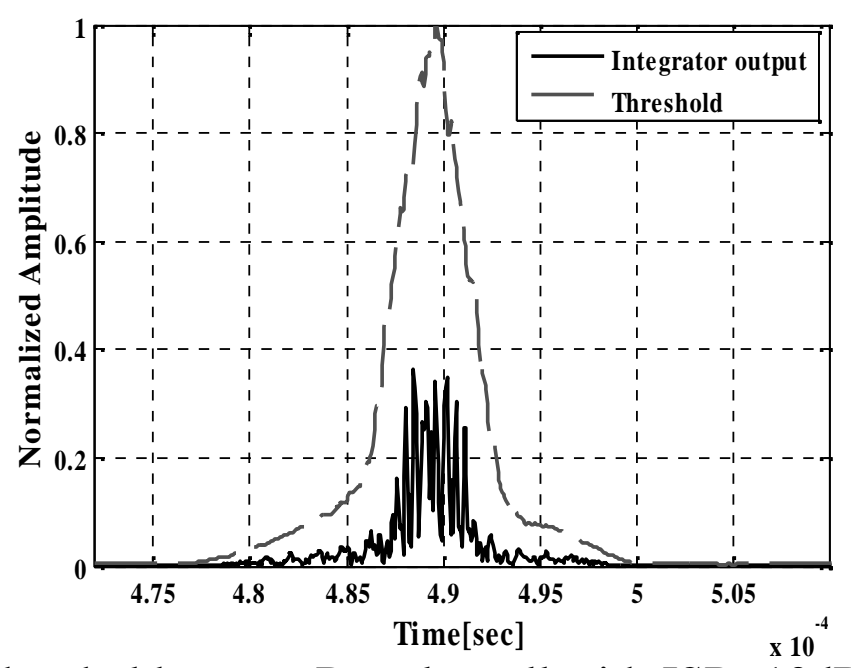

Figure (8): CFAR threshold at zero Doppler cell with JSR=10dB and SNR=-5dB with SOJ or SFJ.

As the input to the CFAR processor is clear from any RFTs at the target Doppler cell, CFAR threshold is not distorted and the target signal crosses the CFAR threshold. In this case, both target signal and CFAR threshold are shown in Figure (10).

To evaluate the effect of chopping jamming on the detection of LFM PC radar quantitatively, detection of the proposed radar model under effect of the modeled chopping jamming is shown in Figure (11) for SOJ or SFJ case and in Figure (12) for SSJ case.

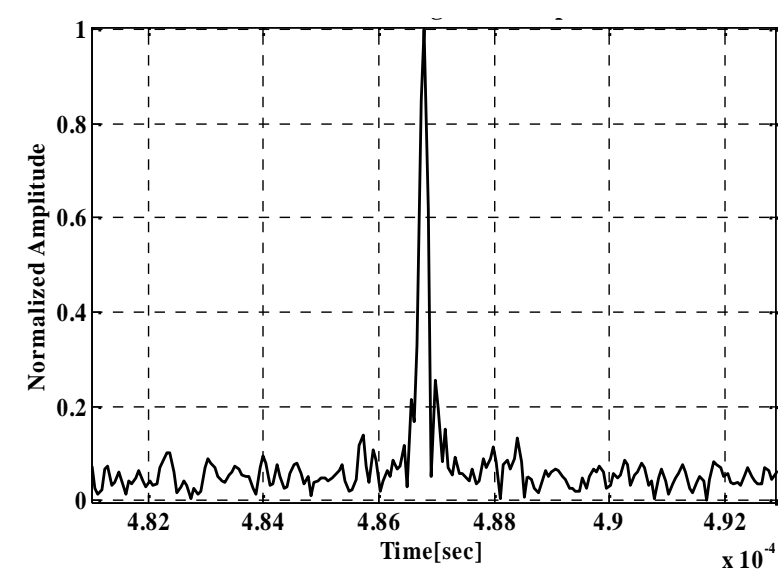

Figure (9): Coherent Integrator output at target Doppler cell with JSR $=10 \mathrm{~dB}$ and $\mathrm{SNR}=-5 \mathrm{~dB}$ with SSJ or SOJ. 


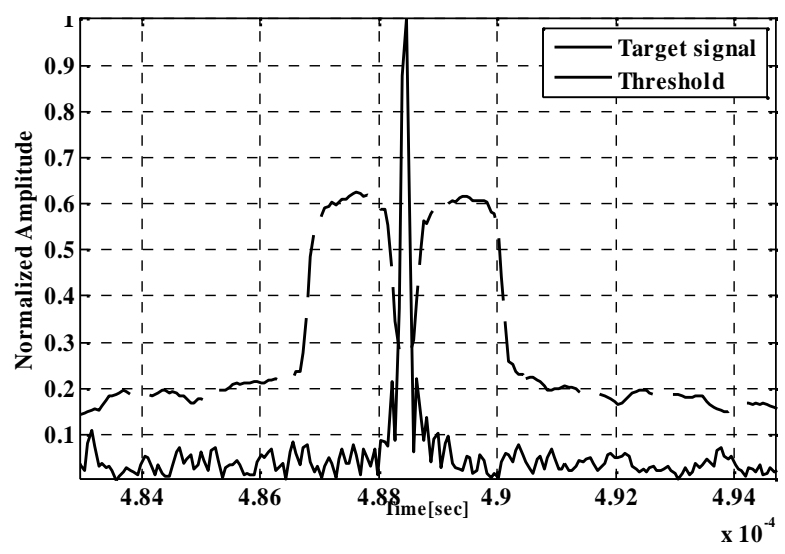

Figure (10): CFAR threshold at JSR $=10 \mathrm{~dB}$ and $\mathrm{SNR}=-5 \mathrm{~dB}$ with SFJ or SSJ.

As expected, the radar detection capability with SFJ or SOJ scenarios has been slightly affected by chopping jamming after passing through coherent integrator even at high JSR. However, chopping jamming by an SSJ has a significant effect on radar detection. As shown in Figure (12), at JSR $=-10 \mathrm{~dB}$ the radar lost its detection at any SNR value. It should be noted that JSR is defined here to be the ratio between the average jamming power and peak signal power. This definition is the classical one used with noise jamming. However, for chopping jamming it is more convenient to consider peak jamming power rather than average jamming power. The ratio between average jamming and peak jamming power is the duty cycle of the jamming waveform which is the same as the duty cycle of the radar waveform for a repeater jammer. For our case, the duty cycle is $6.25 \times 10^{-3}$. This means that the peak jamming power is higher than average jamming power by $22 \mathrm{~dB}$. Hence, the LFM-PC radar lost detection under effect of chopping jamming at peak-to-peak JSR of $12 \mathrm{~dB}$.

In Figure (12), it is noted that the detection curve of the radar without jamming is lower than that with jamming at JSR $=-20 \mathrm{~dB}$ for $\mathrm{SNR}<-22 \mathrm{~dB}$, which is confusing. The reason behind that is the false target generated by chopping jamming at the same target location, which enhances the probability of detection at low SNR up to -22dB. As SNR increases, jamming power is increased to maintain the same JSR ( $-20 \mathrm{~dB}$ here) till the value at which the CFAR threshold is sufficiently raised and the detection ability of the radar is affected. 


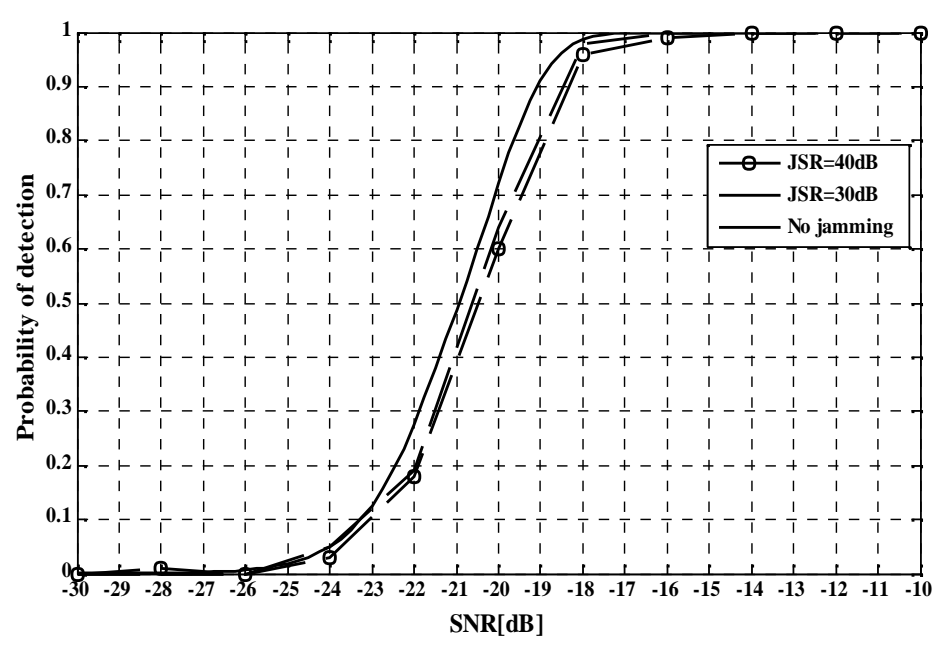

Figure (11): Detection of PC LFM radar in presence of chopping jamming at different JSR with SOJ or SFJ at $\mathrm{P}_{\mathrm{fa}}=10^{-7}$.

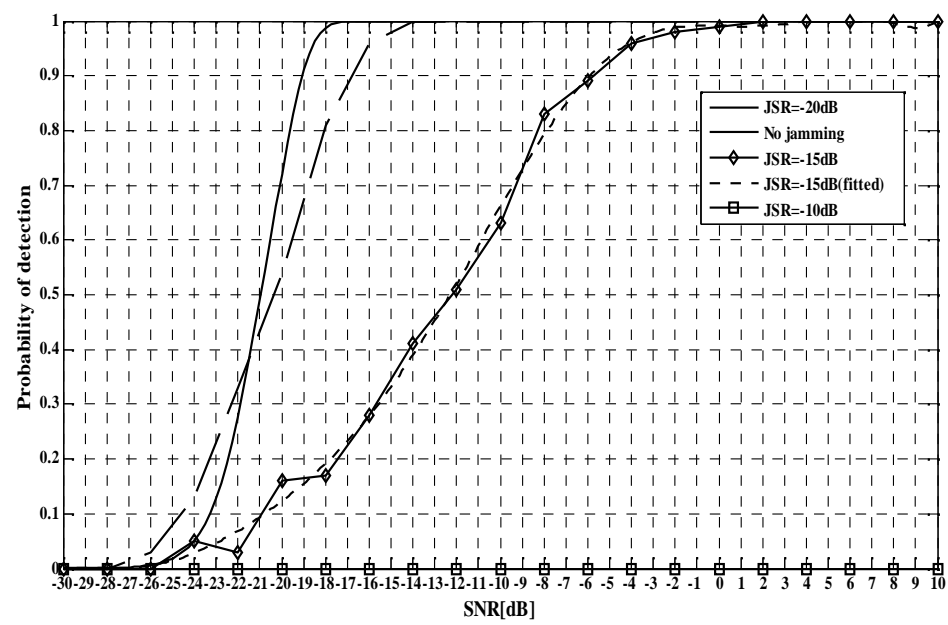

Figure (12): Detection of PC LFM radar in presence of chopping jamming at different JSR with SSJ at $\mathrm{P}_{\mathrm{fa}}=10^{-7}$.

\section{Conclusions:}

Using a verified model, the effect of a simulated chopping jamming on LFM PC radar detection has been investigated. Despite the fact that chopping jamming has been proposed to be used with an SSJ to saturate LFM-PC radar range tracker, it proves a significant effect on radar detection with reasonable JSRs.

When used with an SOJ or SFJ, the detection is slightly affected even at high JSR. The exception occurs when the jammer has prior information about the true target Doppler so that it can generate a jamming signal that covers the corresponding target 11 
Doppler. If the jammer is not an airborne jammer, knowing the target Doppler is not a general case and it increases the complexity of the already complex chopping jamming algorithm.

\section{References:}

[1] M. I. Skolnik, Radar handbook: McGraw-Hill, 1970.

[2] M. Skolnik, Radar Handbook, Third Edition: McGraw-Hill Education, 2008.

[3] C. A. Naval Air Warfare Center Weapons Div Point Mugu, Electronic Warfare and Radar Systems Engineering Handbook: Storming Media, 1997.

[4] M. V. Maksimov, Radar anti-jamming techniques: Artech House, 1979.

[5] W. Fufu, "Simulation Testing of LFM Compression Performances Based on Identification," presented at International Conference on Intelligent System Design and Engineering Application, Changsha, Hunan China, 2010.

[6] G. Galati and E. Institution of Electrical, Advanced Radar Techniques and Systems: Peter Peregrinus, 1993.

[7] Y. Yong, Z. Wen-ming, and Y. Jian-hua, "Study on frequency-shifting jamming to linear frequency modulation pulse compression radars," presented at Wireless Communications \& Signal Processing, 2009. WCSP 2009. International Conference on, 2009.

[8] W. Xue-song and L. Jian-cheng, "Preceded False Target Groups Jamming Against LFM Pulse Compression Radars," in Journal of Electronics \& Information Technology, vol. 30: Science Press, 2008, pp. 1350.

[9] L. G.-l. NIAN Peng-lei, LU Cui-hua,YU Jing, "A Method for Suppressing Convolution Modulation Jamming of LFM Fuze," Telecommunication Engineerin, 2013.

[10] L. Bo, "Simulation study of noise convolution jamming countering to SAR," presented at Computer Design and Applications (ICCDA), 2010 International Conference on, 2010.

[11] M. J. Sparrow and J. Cikalo, "ECM techniques to counter pulse compression radar," Google Patents, 2006.

[12] F. M. A. Ahmed Abu El-Fadl, M. Samir, A. Sisi, "Performance Analysis of Linear Frequency Modulated Pulse Compression Radars under Pulsed Noise Jamming," International Journal of Engineering Research \& Technology, vol. 2, pp. 5, 2013.

[13] N. Levanon and E. Mozeson, Radar Signals: Wiley, 2004.

[14] L. Y. Yibing LI, Yun LIN, "A Method for Sidelobe Suppression of LFM Pulse Compression Signal," Journal of Computational Information Systems, vol. 9, pp. 7, 2013. 
[15] B. R. Mahafza, Radar Systems Analysis and Design Using MATLAB Third Edition: Taylor \& Francis, 2013.

[16] Y. Zhu, G. Zhao, and Y. Zhang, "Research on SAR Jamming Technique Based on Man-made Map," presented at Radar, 2006. CIE '06. International Conference on, 2006.

[17] Z. Wang, M. Gao, Y. Li, and H. Jiang, "Design and application of DRFM system based on digital channelized receiver," presented at Radar, 2008 International Conference on, 2008.

[18] K. Olivier, J. E. Cilliers, and M. du Plessis, "Design and performance of wideband DRFM for radar test and evaluation," Electronics Letters, vol. 47, pp. 824-825, 2011.

[19] C. M. Kwak, "Application of DRFM in ECM for pulse type radar," presented at Infrared, Millimeter, and Terahertz Waves, 2009. IRMMW-THz 2009. 34th International Conference on, 2009.

[20] C. Jiang, M. Gao, Z. Wang, and X. Fu, "Design of High-Speed DRFM System," presented at Computer Science and Information Engineering, 2009 WRI World Congress on, 2009.

[21] Z. Wang, G. Meiguo, L. Yunjie, J. Haiqing, and Y. Sunguo, "The hardware platform design for DRFM system," presented at Signal Processing, 2008. ICSP 2008. 9th International Conference on, 2008.

[22] S. J. Roome, "Digital radio frequency memory," Electronics \& Communication Engineering Journal, vol. 2, pp. 147-153, 1990.

[23] L. B. Van Brunt, Applied ECM: EW Engineering, 1978. 